\title{
Diamond as a magnetic field calibration probe
}

\author{
J Maes ${ }^{1}$, K Iakoubovskii ${ }^{2}$, M Hayne ${ }^{1}$, A Stesmans ${ }^{2}$ and \\ V V Moshchalkov ${ }^{1}$ \\ ${ }^{1}$ Laboratorium voor Vaste-Stoffysica en Magnetisme, K U Leuven, Celestijnenlaan 200 D, \\ B-3001 Leuven, Belgium \\ ${ }^{2}$ Laboratorium voor Halfgeleiderfysica, K U Leuven, Celestijnenlaan 200 D, B-3001 \\ Leuven, Belgium
}

Received 19 January 2004

Published 17 March 2004

Online at stacks.iop.org/JPhysD/37/1102 (DOI: 10.1088/0022-3727/37/7/024)

\begin{abstract}
An optical method is proposed for the calibration of pulsed magnetic fields using photoluminescence from synthetic diamond. Generally, the pulsed magnetic field profile is reconstructed by measuring the pick-up voltage in a small coil with an effective area that needs to be known accurately. A useful method to calibrate this area is presented using the $1.4040 \mathrm{eV}$ optical transition at the $1.4 \mathrm{eV} \mathrm{Ni}$-related centre in diamond. The field value is calculated from the Zeeman splitting of the optical lines using the $g$-factor previously characterized by electron spin resonance. Numerous advantages of the method presented are discussed.
\end{abstract}

\section{Introduction}

\subsection{Some aspects of magnetic field calibration}

A broad range of applications of magnetic measurements underlines the importance of absolute calibration of the magnetic field strength $B$ (Herlach and Miura 2003). Several accurate and reliable procedures are known for calibration of low-field $(B<15 \mathrm{~T})$ dc magnets, such as the magnetic phase transition and the magnetic resonance marker methods. In the first method, a specific magnetic material is placed in the magnet to be calibrated, and the time derivative of magnetization, $\mathrm{d} M / \mathrm{d} t$, is monitored. At certain magnetic field values, $\mathrm{d} M / \mathrm{d} t$ shows discontinuities which can be used for field calibration. The marker method utilizes magnetic level splitting (the Zeeman effect) in a certain magnetic nucleus (the nuclear magnetic resonance probe) or in an electronic defect centre in a solid [the electron spin resonance (ESR) $g$-marker (Stesmans and Van Gorp 1989)]. It relies on the knowledge of the spectroscopic splitting factor $g$ and the ability to measure the resonance frequency. The latter is generally not a limiting factor. So, provided that $g$ is accurately known, a high accuracy calibration can be attained.

In the high-field $(B \sim 60 \mathrm{~T})$ pulsed magnets, a capacitor discharge into a conductive solenoid results in the production of a millisecond-long magnetic field pulse exhibiting a damped sine shape. The field intensity in the coil can be easily monitored using a small metallic (copper wire) pick-up coil, but the absolute field calibration is not straightforward. Generally, an external time-dependent magnetic field $B(t)$ applied parallel to the coil axis induces a voltage over the coil $V(t)=-S_{\text {eff }} \times \mathrm{d} B(t) / \mathrm{d} t$. Here, $S_{\text {eff }}$ is the effective coil area, which takes into account the coil geometry, number of windings and the (small) field inhomogeneity. This parameter is difficult to calculate accurately, and thus should rather be determined from an independent measurement. Unfortunately, both the phase transition and the marker method mentioned above are hardly applicable for this purpose: phase transitions may appear too inertial, while the marker methods would require construction of a complex pulse-field resonance spectrometer solely for the purpose of calibration. Conventionally, such a spectrometer would require a microwave waveguide to be fed into the high-field magnet core, which poses electromagnetic and engineering problem of prohibitive nature. However, most high-field magnets easily allow access via an optical waveguide, thus suggesting following field calibration procedure: a substance should be selected containing a specific paramagnetic centre, that should exhibit an efficient optical transition(s) between an excited state and the ground state. Photoluminescence (PL) transitions should be preferred over optical absorption because of the inherent higher detection sensitivity and easier optical access, i.e. no need to pass light through the sample, meaning that reflection-style geometry with one-way optical access is sufficient and with milder requirements as to the 
Diamond as a magnetic field calibration probe

sample transparency. The magnetic field splitting of the ground and excited states should be described by a simple spin Hamiltonian $H$, preferably of the form $H=g \mu B S$, where $\mu$ is Bohr magneton, and $S$ the $z$-component of the electron spin. Provided that the $g$-factors pertaining to the ground and excited states ( $g_{\text {gr }}$ and $g_{\text {ex }}$, respectively) are accurately calibrated with one of the dc calibration methods described earlier, the pulsed field value can be found from the splitting of the PL transitions as

$$
\Delta E=\mu B\left|S_{\mathrm{gr}} g_{\mathrm{gr}}-S_{\mathrm{ex}} g_{\mathrm{ex}}\right|
$$

The PL measurements can be easily performed with a simple, inexpensive table-top PL spectrometer. Obviously, however, the major difficulty lies in the availability of a proper calibration substance, which should satisfy at least three basic criteria:

(1) It should be mechanically and chemically stable.

(2) It should exhibit an intense and sharp-line PL, easily detectable with high spectral resolution during a short (ms) magnetic field pulse.

(3) It should have significantly different and measurable values of $S_{\mathrm{gr}} g_{\mathrm{gr}}$ and $S_{\mathrm{ex}} g_{\mathrm{ex}}$.

In this respect, we note that efficient electrical dipole allowed transitions usually require $S_{\mathrm{gr}}=S_{\mathrm{ex}}$. Furthermore, it appears that both $g_{\mathrm{gr}}$ and $g_{\mathrm{ex}}$ of many defects in solids are close to the free electron value $g_{\mathrm{e}}=2.002319$, thus resulting in a rather small magnetic field splitting (see equation (1)), and that the desired deviations of $g_{\mathrm{gr}}$ and $g_{\mathrm{ex}}$ from $g_{\mathrm{e}}$ mostly originate from spin-orbit coupling, which strongly increases with the atomic number.

Considering the above criteria and remarks, a most obvious calibration standard for high pulsed magnetic fields would appear a transition metal ion (large spin-orbit coupling) related centre in a transparent, non-magnetic solid, such as $\mathrm{Cr}^{3+}$ in $\mathrm{Al}_{2} \mathrm{O}_{3}$ (ruby). Indeed, ruby is a rather stable technological material, exhibiting very efficient (laser) PL transitions at $\sim 1.79 \mathrm{eV}$. Unfortunately, the ground state of the $\mathrm{Cr}^{3+}$ impurity is a spin triplet exhibiting strong spin-spin interaction $(\sim 0.2 \mathrm{~T})$. This results in a non-linear spin Hamiltonian thus complicating the analysis of the splitting pattern. Therefore, in this paper we propose an alternative standard for the calibration of pulsed magnetic fields, namely the $1.4 \mathrm{eV} \mathrm{Ni}$-related centre in synthetic diamond, which we show is highly suitable for that purpose.

\subsection{Electronic properties of the $1.4 \mathrm{eV} \mathrm{Ni}$-related centre in diamond}

Nickel is one of the most popular catalysts for the growth of diamond using the high-pressure high-temperature (HPHT) technique. It has been found that during the synthesis, dispersed $\mathrm{Ni}$ atoms are incorporated exclusively in the $\{111\}$ growth sectors of diamond, resulting in a number of optical centres. We shall focus here on the sharp lines at 1.4040 and $1.4012 \mathrm{eV}$, which are the dominant features of the so-called $1.4 \mathrm{eV} \mathrm{Ni}$-related centre. The lowest ground state of this centre $\mathrm{gr}_{1}$ (see figure 1) has been characterized by ESR (Isoya et al 1990). It was attributed to an interstitial $\mathrm{Ni}^{+}$related $S=\frac{1}{2}$ centre of trigonal symmetry. The angular dependence

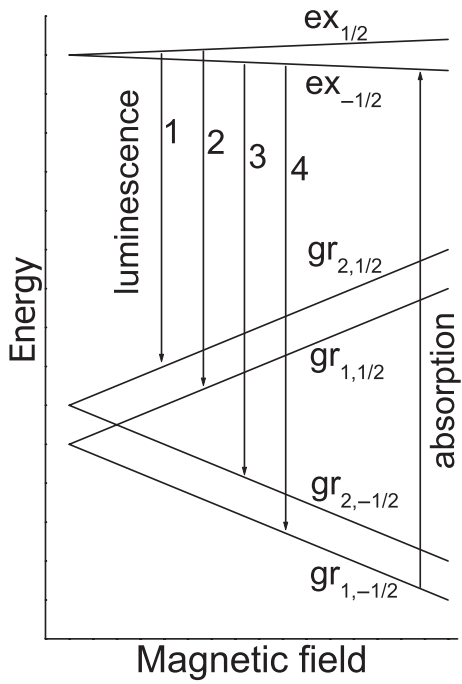

Figure 1. Schematic diagram of some of the optical transitions at the $1.4 \mathrm{eV}$ centre in diamond at helium temperatures with the magnetic field applied along the $\langle 111\rangle$ crystal axis. The ground and excited states are labelled as $\mathrm{gr}_{1, \pm 1 / 2}, \mathrm{gr}_{2, \pm 1 / 2}$ and $\mathrm{ex}_{ \pm 1 / 2}$, respectively, where $\pm 1 / 2$ is the $z$-component of the electron spin.

of ESR lines could be accurately simulated with a simple spin Hamiltonian $H=g \mu B S$. The principal components of the $g$ matrix were deduced as $g_{1 \mathrm{gr} 1}=2.329(5), g_{2 \mathrm{gr} 1}=g_{3 \mathrm{gr} 1}=0$ $(\leqslant 0.1)$, with $g_{1 \mathrm{gr} 1}$ aligned to the [111] crystal axis.

The optical transitions of this $1.4 \mathrm{eV}$ centre have been characterized by optical absorption and luminescence with (Mason et al 1999, Nazare et al 1991) and without (Collins 1989) an applied magnetic field. Those measurements revealed that the 1.4040 and $1.4012 \mathrm{eV}$ lines (energies at zero magnetic field) originate from transition between three Kramers doublets (degenerate only by $\operatorname{spin} S=\frac{1}{2}$ ), one in the excited state $\left(\mathrm{ex}_{ \pm 1 / 2}\right)$ and two, separated by $2.8 \mathrm{meV}$, in the ground state $\left(\mathrm{gr}_{1, \pm 1 / 2}\right.$ and $\mathrm{gr}_{2, \pm 1 / 2}$ in figure 1). All transitions are further split into narrow-spaced $(\sim 0.17 \mathrm{meV})$ multiplets (not shown in figure 1), which were assigned to different $\mathrm{Ni}$ isotopes for a defect involving one $\mathrm{Ni}$ atom. The trigonal symmetry inferred from ESR (Isoya et al 1990) was confirmed by uniaxial stress PL measurements (Nazare et al 1991), and, moreover, an unusual orientational polarization of the $1.4 \mathrm{eV}$ centre was revealed. Normally, a trigonal centre in the diamond lattice has four equivalent orientations. Yet, it is found that within a single (111) growth sector of an HPHT diamond, the $1.4 \mathrm{eV}$ centres are aligned exclusively to the [111], but not to the [11 1$]$, [11ㅣ] or [1111] crystal axes (Collins 1989).

While only the lowest ground state could be characterized by ESR (Isoya et al 1990), the $g$-factors for all three Kramers doublets have been assessed by magneto-optical measurements (Mason et al 1999, Nazare et al 1991). Unfortunately, only relatively low $(\leqslant 6 \mathrm{~T})$ dc magnetic fields were applied in those studies, and as a consequence, the splitting was poorly resolved, resulting in low accuracy. The $g$-values for the excited state were deduced as $g_{1 \mathrm{ex}}=0(\leqslant 0.1), g_{2 \mathrm{ex}}=g_{3 \mathrm{ex}}=$ 2.4(1). The $g_{1}$ value of the higher lying ground state $g_{1 \text { gr2 }}$ was estimated to be in the range 1.6-2 (Mason et al 1999, Nazare et al 1991). In this work, application of higher magnetic fields $(\leqslant 49 \mathrm{~T})$ allowed more accurate determination of the $g_{1 \mathrm{ex}}$ and 


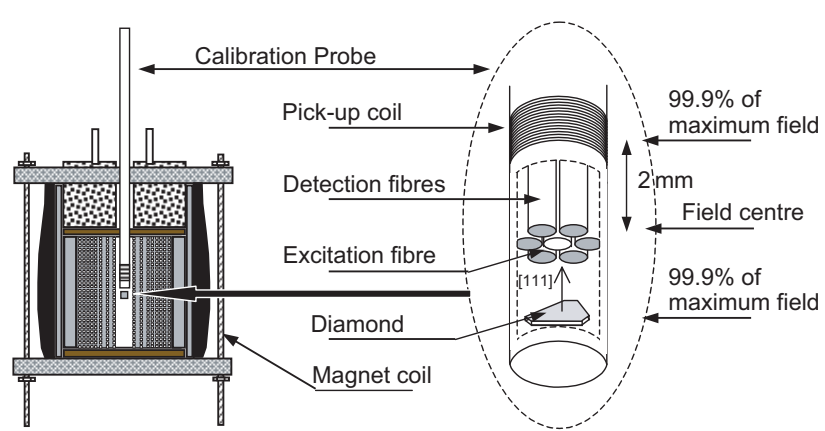

Figure 2. Schematic diagram of the optical probe inside the pulsed field coil.

$g_{1 \mathrm{gr} 2}$ values thus enabling the utilization of the $1.4 \mathrm{eV}$ Ni centre in diamond as a magnetic field calibration standard.

\section{Experimental set-up}

\subsection{Pulsed magnet}

The pulsed magnetic fields were generated using a userfriendly $5 \mathrm{kV}$ capacitor bank with a changeable capacity up to $40 \mathrm{mF}$ and a maximum energy of $600 \mathrm{~kJ}$ (Vanacken 2001). The high-field $(\leqslant 60 \mathrm{~T})$ coils are designed and constructed within the laboratory and are intensively tested according to a precise test-cycle programme (Rosseel et al 2002). As a result of internal reinforcement, i.e. each conductor wire layer is covered by a fibre-composite layer, the coils can withstand on average $>1000$ high-field shots. Consequently, negligible coil degradation occurs during the described experiment. The magnetic field pulse duration is $\sim 20 \mathrm{~ms}$, and the field profile versus time is measured by a pick-up coil positioned in the centre of the magnet coil as depicted in figure 2 . The sample and the pick-up coil are mounted symmetrically $\sim 2 \mathrm{~mm}$ above and below the centre of the pulsed field coil. In this arrangement, the sample and the pick-up coil experience the same magnetic field, equal to $99.9 \%$ of that generated in the centre of the coil.

\subsection{Optical measurements}

In PL measurements the laser light is transmitted by an optical fibre with a core diameter of $200 \mu \mathrm{m}$ down into the He bath cryostat (cryostat not shown in figure 2). Luminescence is collected by six optical fibres surrounding the exciting fibre (see figure 2). Light is dispersed with a compact table-top spectrometer, consisting of a $0.30 \mathrm{~m}$ monochromator equipped with interchangeable gratings and an InGaAs diode array detector. The detector is gated with an electronic shutter triggered by the pick-up coil. Consequently, several accumulations at different magnetic fields can be performed during one magnetic pulse. In this study, however, a single time window of $4 \mathrm{~ms}$ was selected from every pulse with a field variation in time of 5\% (see figure 3 ). This resulted only in a minor broadening of the PL lines that is comparable to or less than the instrumental linewidth of $\sim 0.3 \mathrm{meV}$. The timeintegrated average field during integration was used as the magnetic field value. The magnetic pulse shape and all the

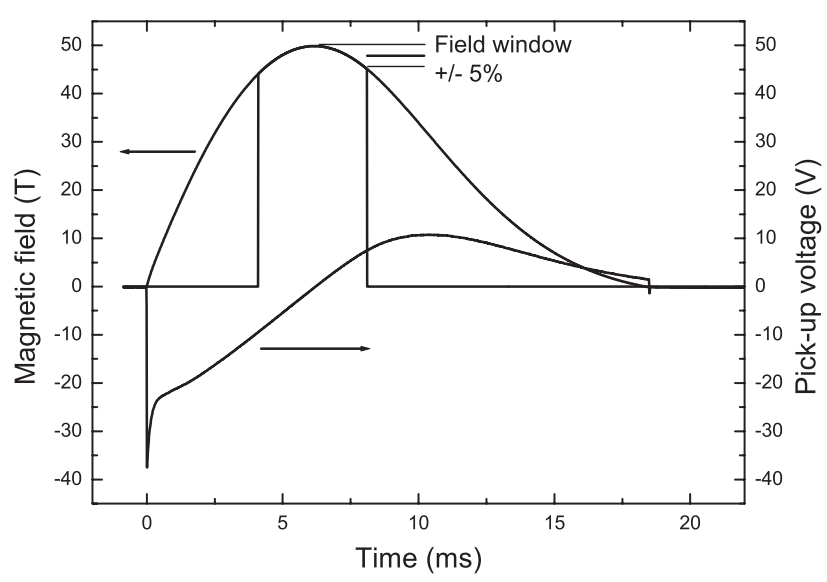

Figure 3. A typical $50 \mathrm{~T}$ pulsed magnetic field profile with the measured pick-up voltage. The shutter opening utilized is indicated by a rectangle.

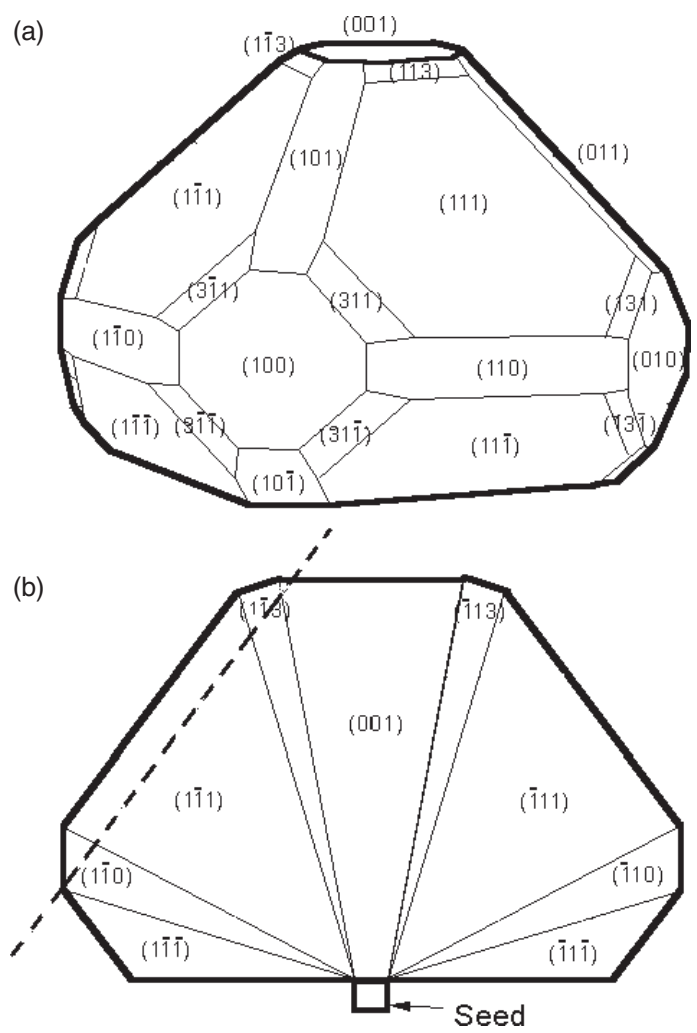

Figure 4. (a) A schematic picture of the HPHT synthetic diamond crystal geometry used in this study with the major growth sectors indicated. This cubo-octahedral morphology is common for HPHT diamond. (b) Bottom picture is a (110) cross-section view of the top crystal. The dashed line marks the (111) plane, along which the initial crystal was cut to produce the calibration sample slice for this study.

triggering pulses remained (almost) identical over the whole range of generated magnetic fields $(0-49 \mathrm{~T})$.

\section{Pulsed field calibration using diamond}

A HPHT diamond exhibiting strong $1.4 \mathrm{eV}$ PL was selected for the field calibration. The diamond had a common cubooctahedral habit with prominent $\{111\}$ facets (see figure $4(a)$ ). 
In order to separate a single $\{111\}$ growth sector, a thin slice was laser cut from a $\{111\}$ facet $[(1 \overline{1} 1)$ facet in figure $4(a)]$, normal to the surface, as indicated by the dashed line in figure $4(b)$. The slice was then polished and mounted into the magnet with the probe fibres and the magnetic field normal to the (111) sample surface (see figure 2). This sample preparation procedure was chosen to simplify the PL spectra and to increase the magnetic splitting (see equation (1)). Indeed, the results summarized in section 1.2 indicate that the $1.4 \mathrm{eV}$ centres are aligned to the [111] direction in a single (111) growth sector, and thus only the $g_{1}$, but not $g_{2}$ and $g_{3}$, components of the $g$ matrices determines the magnetic splitting. Moreover, for the [111] oriented magnetic field $g_{1 \mathrm{ex}}=0$ and $g_{1 \mathrm{gr} 1}$ reaches its maximum value (2.329), thus maximizing $\Delta E$ (see equation (1)).

PL measurements were performed at $4.2 \mathrm{~K}$ using $2.41 \mathrm{eV}$ laser excitation from an argon-ion laser. Typical results are summarized in figure 5, where part $(a)$ shows typical PL spectra. The derived peak positions as a function of magnetic field are plotted in figure $5(b)$, and the integrated intensities of lines 1 and 4, as well as of the total PL spectrum, are shown in figure $5(c)$. Lines 2 and 3 were only partially resolved for most magnetic field values assessed and therefore, to provide reliable data, a half-sum of their intensities is plotted in figure $5(c)$. The triangular-like lineshape of the signals shown in figure $5(a)$ is caused by the unresolved $\mathrm{Ni}$ isotopic splitting of each line (the major $\mathrm{Ni}$ isotopes have natural abundances of $68.27 \%$ for ${ }^{58} \mathrm{Ni}, 26.1 \%$ for ${ }^{60} \mathrm{Ni}$ and $3.59 \%$ for ${ }^{62} \mathrm{Ni}$ ). Calibration of the magnetic field was achieved by matching the slopes for lines 2 and 4 (figure $5(b)$ ) to the values $S_{\mathrm{gr} 1} g_{1 \mathrm{gr} 1} \mu$, where $g_{1 \mathrm{gr} 1}=2.329$ as previously deduced by ESR (Isoya et al 1990) and $S_{\mathrm{grl}}= \pm \frac{1}{2}$. Concomitantly, the $g$ value for the second ground state (lines 1 and 3) was determined here as $g_{1 \mathrm{gr} 2}=1.93(2)$. It is important to note that this calibration relies on the assumption $g_{1 \mathrm{ex}}=0$, which will be justified in section 4.

The calibration procedure described requires detection of the magnetic field splitting between lines 2 and 4, for which purpose a particular laser-cut slice was prepared in this study. However, measurements performed before the sample was cut revealed that although the splitting pattern from the whole diamond crystal is more complex than that from a (111) slice, lines 2 and 4 could still be clearly separated. Therefore, a whole as-grown HPHT crystal can be used for the proposed calibration method. Moreover, the $1.4 \mathrm{eV}$ lines are sufficiently sharp even at nitrogen temperatures (linewidth $\sim 0.8 \mathrm{meV}$ at $77 \mathrm{~K}$ ), and thus the calibration procedure does not even require a He cryostat.

\section{Analysis}

The proposed magneto-optical marker calibration method relies on the knowledge of the values $g_{1 \mathrm{gr} 1}$ and $g_{1 \mathrm{ex}}$ (see equation (1)). While $g_{1 \mathrm{gr} 1}$ for the $1.4 \mathrm{eV}$ centre was previously accurately determined by ESR as 2.329(5), only a rough estimate was available for $g_{1 \mathrm{ex}}$. In this work, application of low temperatures and high magnetic fields allowed us to increase the accuracy of the $g_{1 \mathrm{ex}}$ value by an order of magnitude. This is attained starting from the analysis of figure 5(c), where, clearly, two regions, 0-25 and 25-49 T, can be distinguished: a key observation is that the line intensities
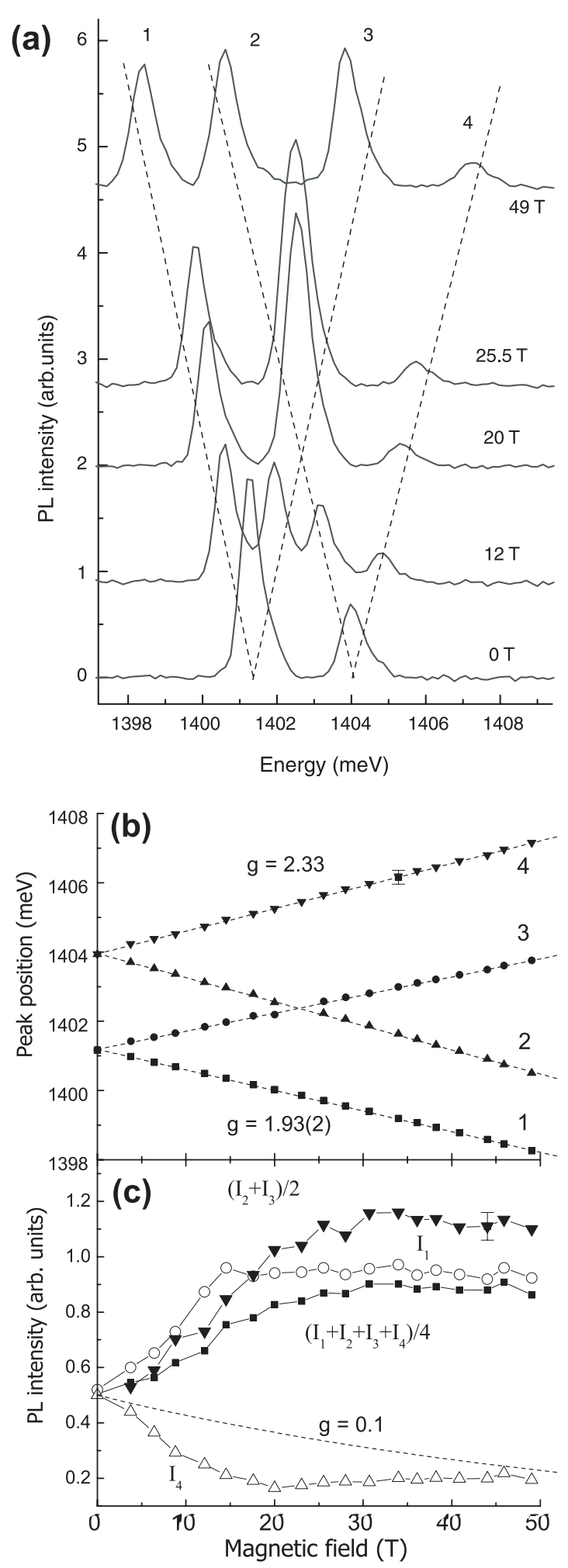

Figure 5. Magneto-PL data obtained at $4.2 \mathrm{~K}$ under $2.41 \mathrm{eV}$ laser excitation with the pulsed magnetic field applied along a $\langle 111\rangle$ crystal axis: (a) PL spectra for selected field values; dashed lines indicate the splitting behaviour as a function of magnetic field. (b) Peak positions; dashed lines present linear fits to the data based on the Zeeman splitting $g \mu B S_{Z}$. (c) Intensities of the observed lines and some combinations as a function of the magnetic field. Solid lines in $(c)$ are guides for the eye, while the dashed line depicts the expected thermalization behaviour of the PL intensity for $g_{1 \mathrm{ex}}=0.1$ (see text for details). 
versus magnetic field remain constant in the second region. Indeed, the magnetic splitting in the excited state of the $1.4 \mathrm{eV}$ centre may cause electron thermalization thus resulting in a (slight) intensity increase for lines 3 and 4, which would result in a corresponding intensity decrease for lines 1 and 2 (see figure 1). The expected effect on the intensity of lines 1 and 2 of such a thermalization behaviour, corresponding to the magnetic splitting with $g_{1 \mathrm{ex}}=0.1$ (upper limit given by Mason et al 1999), is simulated and presented by the dashed line in figure $5(c)$. The experimental data, however, show no significant intensity variation in the field range $25-49 \mathrm{~T}$. This suggests that the splitting in the excited state is rather small, from where it is inferred that $g_{1 \mathrm{ex}}<0.01$. The latter improved estimate justifies, within the current accuracy, the assumption $g_{1 \mathrm{ex}}=0$ made in the calibration procedure outlined in section 3 .

In the first region $(0-25 \mathrm{~T})$ in figure $5(c)$, the intensities of the lines 1 and $(2+3)$ increase, but the intensity of the line 4 decreases. This behaviour can be partially explained by self-absorption, as described below (see figure 1): at the low temperature of our experiment $(4.2 \mathrm{~K})$ only the lowest ground state $\mathrm{gr}_{1}$ is populated. Therefore, at zero magnetic field, $\mathrm{PL}$ originating from transitions $\mathrm{ex}_{ \pm 1 / 2} \rightarrow \mathrm{gr}_{1, \pm 1 / 2}$ (PL lines 2 and 4), but not $\mathrm{ex}_{ \pm 1 / 2} \rightarrow \mathrm{gr}_{2, \pm 1 / 2}$ (PL lines 1 and 3), is partially absorbed via transitions $\mathrm{gr}_{1, \pm 1 / 2} \rightarrow \mathrm{ex}_{ \pm 1 / 2}$. Note that optical dipole transitions $\mathrm{gr}_{1,+1 / 2} \rightarrow \mathrm{ex}_{-1 / 2}\left(\right.$ or $\mathrm{gr}_{1,-1 / 2} \rightarrow$ $\mathrm{ex}_{+1 / 2}$ ) are spin forbidden and thus can be neglected here. Increasing magnetic field results in the splitting of the level $\mathrm{gr}_{1}$ into $\mathrm{gr}_{1,+1 / 2}$ and $\mathrm{gr}_{1,-1 / 2}$. Consequently, electrons thermalize into the level $\operatorname{gr}_{1,-1 / 2}$. As a result, the absorption transition $\mathrm{gr}_{1,-1 / 2} \rightarrow \mathrm{ex}_{-1 / 2}$ is enhanced (and is drawn in figure 1) and that of $\operatorname{gr}_{1,1 / 2} \rightarrow \mathrm{ex}_{1 / 2}$ is quenched. Correspondingly, the PL transition 4 (2) becomes more (less) self-absorbed. Note, that this simple model would naturally explain the intensity decrease of PL line 4 and increase of line 2 in figure 5(c), but not the intensity variations of lines 1 and 3 . The origin of the latter variations is yet unclear and requires further investigation.

\section{Conclusion}

In conclusion, we have developed a simple method for calibration of pulsed magnetic fields. The field is continuously measured by a voltage across a small pick-up coil inserted into the magnet. This voltage is calibrated utilizing the Zeeman splitting of the $1.4040 \mathrm{eV}$ PL transition at the $1.4 \mathrm{eV}$ Ni-related centre in synthetic diamond, the splitting being measured with magnetic field along a $\langle 111\rangle$ crystal axis. Application of high magnetic fields (49 T) along a [111] direction and of low temperatures $(4.2 \mathrm{~K})$ in this study allowed accurate determination of the $g$ values at the $1.4 \mathrm{eV}$ centre: $g_{1 \mathrm{gr} 2}=1.93(2)$ for the second, higher-lying ground state, and $g_{1 \mathrm{ex}}<0.01$ for the excited state.

\section{Acknowledgments}

The authors are grateful to $\mathrm{H}$ Kanda (NIMS, Japan), I Kiflawi (Reading University, UK) and A Taylor (DTC Research Centre) for preparation and provision of the diamond sample. KI acknowledges illuminating discussions with Prof. J M Baker. This work has been supported by the FWO-Vlaanderen, the Belgian IUAP programmes, and the VIS 00/001 project of the K U Leuven and the SBO-project 30219 of the IWT-Vlaanderen.

\section{References}

Collins A T 1989 J Phys.: Condens. Matter 1439

Herlach F and Miura N 2003 High Magnetic Fields: Science and Technology (Singapore: World Scientific) ISBN 9810246986

Isoya J, Kanda H and Uchida Y 1990 Phys. Rev. B 429843

Mason P W, Ham F S and Watkins G D 1999 Phys. Rev. B 605417

Nazare M H, Neves A J and Davies G 1991 Phys. Rev. B 4314196

Rosseel K, Lagutin A, Herlach F, Boon W, Bruynseraede Y and Van Humbeeck J 2002 IEEE Trans. Appl. Supercond. 12707

Stesmans A and Van Gorp G 1989 Rev. Sci. Instrum. 602949

Vanacken J 2001 Physica B 294-295 591 